\title{
Surgical approaches for treatment of ureteropelvic junction obstruction - a systematic review and network meta- analysis
}

Annemarie Uhlig ${ }^{1 *}$ (D) Johannes Uhlig ${ }^{2,3}$, Lutz Trojan ${ }^{1}$, Marc Hinterthaner ${ }^{4}$, Alexander von Hammerstein-Equord ${ }^{4+}$ and Arne Strauss $^{1+}$

\begin{abstract}
Background: Multiple surgical treatment options are available for the treatment of ureteropelvic junction obstruction (UPJO). The aim of this study is to compare the most frequently used technics in a comprehensive network approach.

Methods: A systematic literature search of the EMBASE, MEDLINE and COCHRANE libraries was conducted in January 2018. Publications were included that evaluated at least two of the following surgical techniques: open pyeloplasty (OP), endopyelotomy (EP), laparoscopic (LP) and robot assisted pyeloplasty (RP). Main outcomes were operative success, complications, urinary leakage, re-operation, transfusion rate, operating time, and length of stay. Network meta-analyses with random effects models simultaneously assessed effectiveness of all surgical techniques.

Results: A total of 26 studies including 3143 patients were analyzed. Compared with RP, EP and LP showed lower operative success rates (EP: OR $=0.09,95 \% \mathrm{Cl}: 0.05-0.19 ; p<0.001 ; \mathrm{LP}: \mathrm{OR}=0.51,95 \% \mathrm{Cl}: 0.31-0.84 ; p=0.008$ ). Compared with OP, LP and RP had lower risk for complications (LP: OR=0.62; 95\%Cl:0.41-0.95; $p=0.027$; RP: OR= $0.41 ; 95 \% \mathrm{Cl}: 0.22-0.79 ; p=0.007)$. Compared with RP, no significant differences were detected for urinary leakage or re-operation, transfusion rates. Compared with EP, RP yielded longer operating time (mean $=102.87 \mathrm{~min}, 95 \% \mathrm{Cl}$ : 41.79 min-163.95 min, $p=<0.001$ ). Further significant differences in operating times were detected when comparing LP to EP (mean $=115.13 \mathrm{~min}, 95 \% \mathrm{Cl}: 65.63 \mathrm{~min}-164.63 \mathrm{~min}, p=<0.001)$ and OP to EP (mean $=91.96 \mathrm{~min}$, 95\%Cl:32.33 min-151.58 min, $p=0.003)$.

Conclusions: Multiple surgical techniques are available for treatment of UPJO. RP has the highest rates of operative success and as well as LP lower complication rates than OP. Although surgical outcomes are worse for EP, its operating time is shorter than OP, RP, and LP. Surgeons should consider these findings when selecting the optimal treatment method for individual patients.
\end{abstract}

Keywords: Endoscopy, Laparoscopy, Minimally invasive surgical procedures, Laparotomy, Network meta-analysis

\footnotetext{
* Correspondence: annemarie.uhlig@med.uni-goettingen.de

${ }^{+}$Alexander von Hammerstein-Equord and Arne Strauss contributed equally

to this work.

'Department of Urology, University Medical Center Goettingen, Goettingen,

Germany

Full list of author information is available at the end of the article
}

(c) The Author(s). 2019 Open Access This article is distributed under the terms of the Creative Commons Attribution 4.0 International License (http://creativecommons.org/licenses/by/4.0/), which permits unrestricted use, distribution, and reproduction in any medium, provided you give appropriate credit to the original author(s) and the source, provide a link to the Creative Commons license, and indicate if changes were made. The Creative Commons Public Domain Dedication waiver (http://creativecommons.org/publicdomain/zero/1.0/) applies to the data made available in this article, unless otherwise stated. 


\section{Background}

Surgical techniques for treatment of ureteropelvic junction obstruction (UPJO) have seen significant advancements during the last decades. From open approaches over laparoscopic techniques and endopyelotomy (EP) in the 1990s, robot assisted approaches have been introduced in 2002 [1, 2]. Currently, all of these techniques are clinically applied, although laparoscopic and robot assisted pyeloplasty (LP and RP) are often claimed to be superior to other approaches [3-7]. Several studies reported that minimal invasive treatment options outperform open pyeloplasty (OP) with respect to early recovery and lower complication rates, whereas OP operating time is shorter $[3,4]$. Comparing LP and RP, length of hospital stay, operating and suturing time seem to be shorter for RP [8-11]. In one meta-analysis, operative success rate was significantly higher for RP [9]. While abdominal surgery is required for open, laparoscopic and robot assisted approaches, EP probably has the lowest invasiveness [12]. Even though EP has been replaced by other approaches in many institutions, several experts in the field still advocate this technique [13]; especially, as few literature is available summarizing the evidence on all approaches. Notwithstanding that metaanalyses have been published comparing individual studies for two treatment options, these provides limited guidance in the current situation with multiple approaches available for UPJO which must be evaluated against each another. The aim of this network-meat analysis is to provide a comprehensive overview for most frequently used techniques for treatment of UPJO and to compare their effectiveness regarding various clinically important outcomes.

\section{Materials and methods \\ Search strategy}

This study was registered a priori at PROSPERO (CRD42018085917). The systematic literature search using EMBASE, MEDLINE and COCHRANE libraries was performed in January 2018 and was unrestricted with respect to publication language and date. In addition, publication lists of reviews and included articles as well as conference proceedings were searched. Furthermore, we searched a registry for clinical trials (www.clinicaltrials. gov) to identify potentially unpublished studies. The full search strategy is available in the Additional file 1.

\section{Criteria for study inclusion and exclusion}

We included retro- and prospective studies comparing at least two of the following surgical approaches for the treatment of UPJO: OP, LP, RP and EP. Studies had to assess at least one of the following outcomes: operative success, operating time, suturing time, estimated blood loss, transfusion rate, conversion rate, re-operation rate, intra- and postoperative as well as overall complications, urinary leakage, deaths, length of hospital stay, time to return to normal activities, renal function, pain, analgesia requirement, survival time and costs. No minimum follow-up time was required for study inclusion. Of these, the following outcomes were statistically evaluated: operative success was examined as the total number of successes as defined by the authors; rates of transfusion, conversion, re-operation as well as intraand postoperative complications, and urinary leakage were evaluated as total numbers as well. Reviews and meta-analyses were excluded as well as studies focusing on children or animals. In addition, we excluded studies which reported insufficient data on measures of dispersion or pooled outcome data of two surgical approaches. In case of more than one publication reporting on the same patient cohort, the more comprehensive one was selected in order to meet the assumption of independence for meta-analyses.

\section{Definition of operative success}

The authors of the papers used varying definitions for operative success: Objective success was reported by all authors and included mostly included patent ureteropelvic junction confirmed by radionuclide diuretic renogram or intravenous urography (IVU) and sometimes decrease in severity of hydronephrosis. Objective success was often defined as absence of symptoms or "significant" improvement with no further specification. If separate measures for subjective and objective success were reported instead of combined values, only success rates of objective measures were included in the in the statistical analysis to account for the subjectivity of perceived pain [14-16]. Measures for success of the surgical procedure e.g. whether a laparoscopic surgery could be performed without conversion, were not taken into account.

\section{Data extraction}

Publication titles identified via the literature search were independently screened by 3 blinded authors resulting in a selection for abstract and full text screening which was performed by 2 independent blinded authors. From the resulting list of publications suited for inclusion, data extraction was performed in the same manner. Disagreement was resolved by consultation of a third author and majority vote. As suggested by Rothman et al., a study was considered as prospective if data collection on interventions and covariates took place before the outcome occurred [17].

\section{Assessment of study quality}

Study quality assessment using the Downs and Black instrument was performed by two independent blinded authors resolving disagreement by consensus involving a 
third author [18]. The Downs and Black instrument rates the quality of randomized and non-randomized studies on a scale from 0 to 32 points $(0$ points for the worst and 32 for the best study quality) using a catalogue of 27 items. For each item 1 point is given except for the description of the distribution of principal confounders in each group of subjects where a maximum of 2 points can be reached and for the evaluation of study power for which a maximum of 5 points can be reached. For power evaluation study sizes were credited 1 up to 5 points for $<15,15$ to 44,45 to 59,60 to 100 , > 100 patients according to the quartiles of sample sizes of included studies. Study quality was labeled "low" (1-10 points on Downs and Black instrument), "moderate" (11-21 points) and "high" (22-32 points). The Newcastle Ottawa Scale was used for additional study quality assessment. As suggested by the Cochrane handbook, study quality was separately assessed for each outcome [19].

\section{Statistical analyses}

For dichotomous outcomes, Odds Ratios (OR) were calculated from absolute numbers or percentage given in standard manner. Continuous outcomes such as operating time, estimated blood loss and length of hospital stay were compared as median or mean with standard deviation. If available, data from multivariable models was preferentially used [20, 21]. In case of two treatment for similar interventions e.g. endopyelotomy and acucise endopyelotomy, the results were pooled [22]. If only median and interquartile range (IQR) for continuous outcomes were reported, and a large sample size indicated the high probability of an underlying normal distribution, the standard deviation was calculated by dividing the IQR by 1.35 [16]. All outcomes were compared to endopyelotomy as the reference group. A network metaanalysis with random effects approach was used as statistical method for comparison. All outcomes were ranked by p-score methods to estimate the amount of certainty that a single treatment outperforms the average of competing interventions. The $\mathrm{p}$-score ranges from 0 to 1 , the latter indicating the highest certainty possible [23]. Study heterogeneity was evaluated by Higgins's I ${ }^{2}$ considering percentages below $25 \%$ as of potentially low relevance, from 26 to $50 \%$ as "moderate", from 51 to $75 \%$ as "substantial", and from 76 to $100 \%$ as "considerable" heterogeneity [19]. The consistency assumption was evaluated via visual assessment of net heat plots and by Cochran's Q statistic. All pairwise comparisons of more than 10 studies were tested for publication bias using the weighted linear regression of the treatment effect on its standard error [24]. Sensitivity analyses were performed including only studies with at least 12 months follow-up time. The statistical analysis was performed using $\mathrm{R}$ version 3.4.2 with the packages "meta", "netmeta", and "metabias" as well as $\mathrm{R}$ Studio version 1.1.383. All $p$-values were calculated two-sided and an alpha-level of $<0.05$ was considered statistically significant.

\section{Results \\ Study characteristics}

The systematic literature search yielded 3008 studies published between 1995 and 2017 of which 26 fulfilled the inclusion criteria [14-16, 20-22, 25-44]. Figure 1 depicts the selection process of the studies whereas Table 1 details the study characteristics. In total, 3143 patients were analyzed: 556 receiving OP, 1540 receiving LP, 798 receiving RP, and 249 receiving EP. Operative success and complications were evaluated by 24 studies each, whereas operating time was reported by 22 studies and length of hospital stay by 21 studies. Estimated blood loss was evaluated by 13 studies, postoperative complications by 10 studies, conversion rates by 9 studies and re-operation rates by 8 studies. Transfusion rates, intraoperative complications, urinary leakage and analgesia requirement were reported by 7 studies each. Four studies evaluated death rates, whereas suturing time and time to return to normal activities was reported by 3 studies each. Pain, renal function, and costs were evaluated by 1 study each whilst none of the included studies reported on survival time. Six studies were three-armed trials $[14,21,25,28,30,40]$; the remaining 20 studies evaluated two of the 4 outcomes. Figure 2 details the number of comparisons for each end point. Only 3 congress abstracts provided sufficient data to meet the inclusion criteria $[30,31,38]$. Study design was retrospective in 18 studies, 1 study included preand retrospective data [40] and 5 studies explicitly stated a prospective design [27, 36, 38, 41, 44]. For 2 studies no information was available [31,32]. Only one study had a randomized controlled design [38] and two further studies used multivariable adjustment methods to account for confounding [20, 21]. The mean follow up ranged from 1 month to greater than 6 years with the majority of the studies reporting follow-up times greater than 1 year. The study population consisted of adult patients in 15 of the included publications, whereas 8 studies reported on mixed cohorts of adults and children with the majority of patients being of age $>18$ years in case of available information [30,32-34, 41-44]. For 3 studies the inclusion criteria with respect to patient age were unclear $[21,31,38]$. The percentage of female patients ranged from 32 to $77 \%$ with 6 studies omitting this information $[15,16,25,26,31,38]$. The geographic region of the study population was Asia in 8 cases [22, 30, 32, 34, 37, 40, 41, 43], Europe for 7 studies [15, 29, 31, 33, $35,38,42]$, and North America for 10 studies [16, 20, $21,25-28,36,39,44]$. One study reported on a mixed Asian and North American population [14]. 


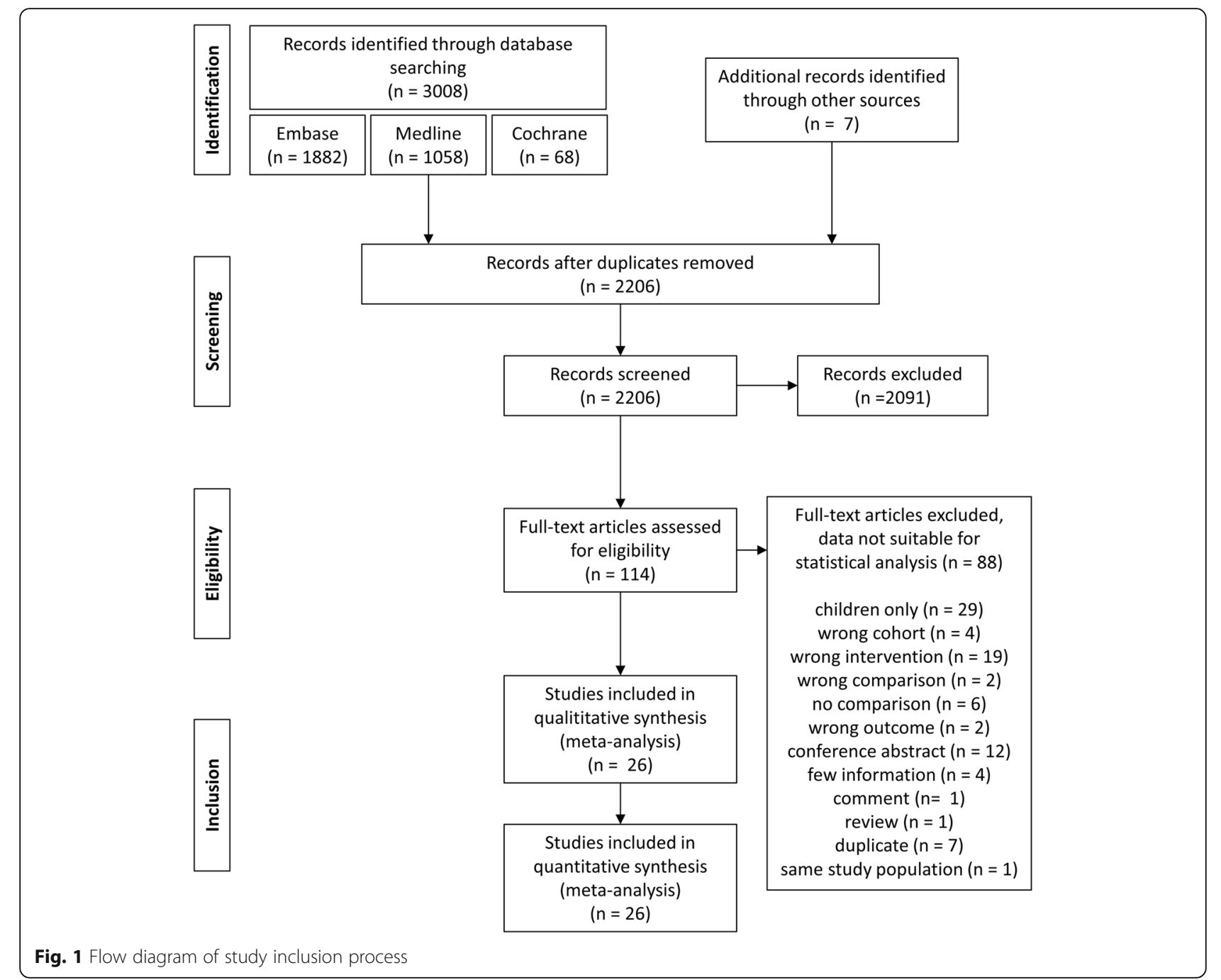

\section{Study quality}

The median study quality was 14 points (range from 6 to 25 points). Reasons for the moderate quality were missing randomization, allocation concealment and blinding as well insufficient confounder adjustment and loss to follow-up in almost all studies. Nevertheless, many studies clearly described study hypothesis, main outcomes and findings as well as patient characteristics and selected participants representative for the source population. Table 2 details the study quality separately for the most important endpoints success and complications. Comparable results were obtained using the Newcastle Ottawa scale (Additional file 1: Table S3).

\section{Network meta-analyses for different outcomes Network meta-analysis of operative success}

The analysis of operative success included 34 pairwise comparisons from 24 studies. Compared with RP, EP and LP showed lower success rates with $\mathrm{OR}=0.09$ $(95 \% \mathrm{CI} 0.05-0.19 ; p<0.001)$ for $\mathrm{EP}$ and $\mathrm{OR}=0.51$
(95\%CI0.31-0.84; $p=0.008)$ for LP. No statistically significant difference was evident comparing OP and RP $(\mathrm{OR}=0.69,95 \% \mathrm{CI}$ 0.34-1.4; $p=0.306)$. Table 3 depicts all pairwise comparisons in a league table. Associated pscores are presented in Table 4. There was no evidence of study heterogeneity $\left(\mathrm{I}^{2}=0 \%, p=0.9041\right)$. Neither Cochran's $\mathrm{Q}(\mathrm{Q}=1.98 ; p=0.9213)$ nor the net heat plot depicted inconsistencies. Comparisons for OP, RP, LP, and EP as forest-plot are shown in Fig. 3a. Upon sensitivity analyses including only those studies with at least 12 months follow-up time $(n=13)$, comparable results were obtained (Additional file 1: Table S1). A total of 15 studies provided information on primary versus secondary UPJO [14, 16, 21, 22, 25, 27-29, 35-37, 39-41, 44]. Of these, only 9 studies explicitly included secondary UPJO [16, 21, 22, 25, 27, 28, 35, 37, 44]: Only 2 studies compared operative success between primary and secondary UPJO [25, 30]. Baldwin et al. stated, that for LP operative success was higher in the group with secondary UPJO (100\% vs $89 \%)$. But for EP operative success 


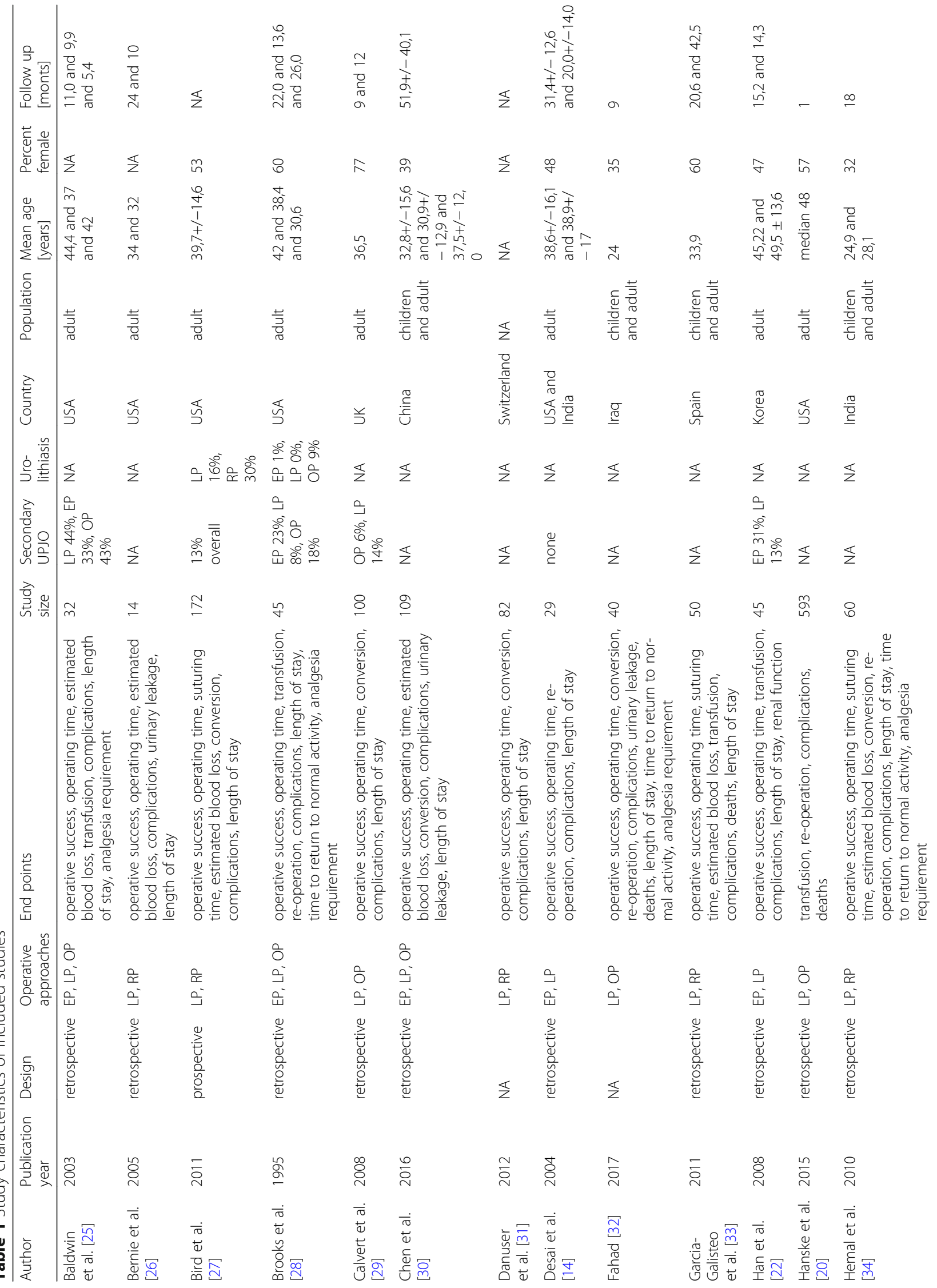




\begin{tabular}{|c|c|c|c|c|c|c|c|c|c|c|c|c|c|}
\hline 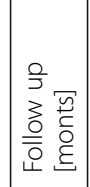 & 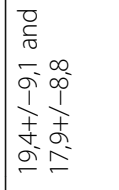 & $\begin{array}{l}\mathrm{O} \\
\mathrm{in}\end{array}$ & 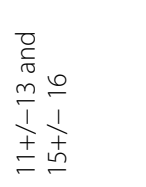 & 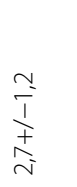 & $\stackrel{\sim}{\wedge}$ & 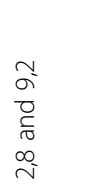 & 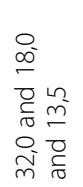 & $\stackrel{\text { In }}{\sigma}$ & 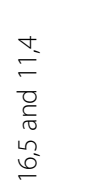 & 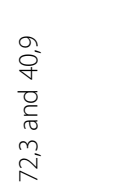 & 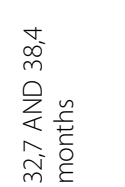 & $\begin{array}{l}0 \\
\frac{0}{0} \\
\frac{0}{0} \\
0\end{array}$ & 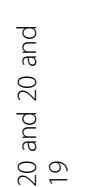 \\
\hline 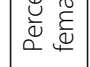 & 8 & $\stackrel{\llcorner}{0}$ & 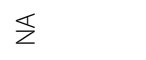 & or & $\mathbb{z}$ & in & $\stackrel{m}{m}$ & $\bar{z}$ & $\stackrel{\infty}{m}$ & in & $\stackrel{+}{m}$ & in & $\bar{\sigma}$ \\
\hline 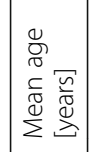 & 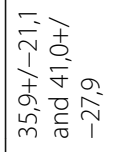 & 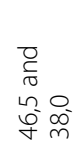 & $\stackrel{n}{m}$ & $\begin{array}{l}\stackrel{\rho}{m} \\
\stackrel{0}{c} \\
\substack{0 \\
\infty \\
\sim}\end{array}$ & $\Sigma$ & $\begin{array}{l}\overline{0} \\
\bar{c} \\
\overline{0} \\
m \\
\sigma^{\infty} \stackrel{\infty}{m}\end{array}$ & 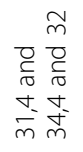 & 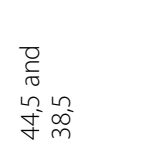 & 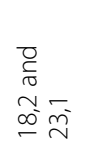 & $\mathscr{F}$ & 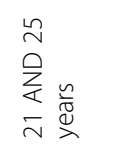 & $\begin{array}{l}\stackrel{n}{2} \\
\stackrel{N}{1} \\
0 \\
\stackrel{0}{0} \\
\stackrel{0}{0}\end{array}$ & $\stackrel{\$}{z}$ \\
\hline $\begin{array}{l}\frac{c}{0} \\
\frac{0}{0} \\
\frac{0}{0} \\
\frac{0}{0}\end{array}$ & $\frac{n}{\frac{5}{0}}$ & $\frac{n}{\frac{H}{0}}$ & $\underline{z}$ & $\frac{n}{\frac{n}{5}}$ & 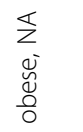 & 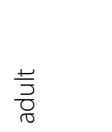 & $\frac{\sqrt[n]{5}}{\frac{5}{0}}$ & 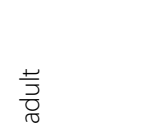 & 离 & 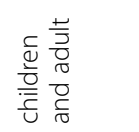 & 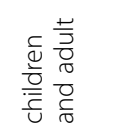 & 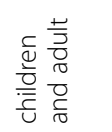 & $\frac{\sqrt{5}}{\frac{5}{0}}$ \\
\hline 突 & 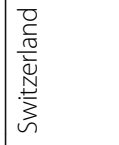 & 壱 & 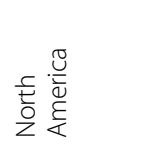 & 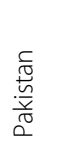 & 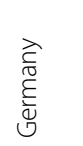 & 芯 & $\underline{\frac{\pi}{\overline{0}}}$ & 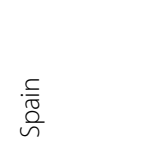 & 胥 & 离 & 异 & 崩 & 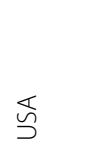 \\
\hline 它 & $\stackrel{\nwarrow}{z}$ & $\Sigma$ & 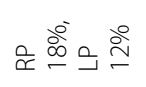 & z & $\Sigma$ & $\S$ & $\Sigma$ & & $\frac{\pi}{z}$ & & $\mathbb{z}$ & $\Sigma$ & $\Sigma$ \\
\hline 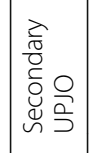 & $\begin{array}{l}0 \\
0 \\
\infty \\
\infty \\
0 \\
9 \\
0\end{array}$ & $\stackrel{\mathscr{c}}{0}$ & 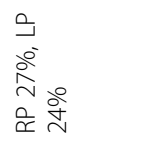 & $\stackrel{\mathscr{0}}{\check{0}}$ & $\stackrel{\Sigma}{z}$ & $\stackrel{0}{0}$ & $\stackrel{\check{\sigma}}{0}$ & $\stackrel{\$}{z}$ & $\stackrel{\mathscr{0}}{\circ}$ & $\mathbb{z}$ & 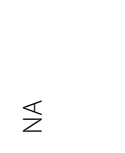 & $\begin{array}{l}\frac{a}{a} \\
\dot{a}^{\circ} \\
\text { a do }\end{array}$ & 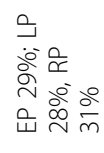 \\
\hline 总. & 夏 & $\stackrel{i}{ }$ & 产 & $\stackrel{n}{n}$ & 㶽 & ণ & 8 & $\alpha$ & o & g & $\stackrel{\mathbb{E}}{ }$ & 号 & 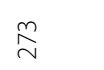 \\
\hline $\begin{array}{l}\frac{n}{c} \\
\frac{\partial}{0} \\
\frac{\partial}{0} \\
\frac{0}{4}\end{array}$ & 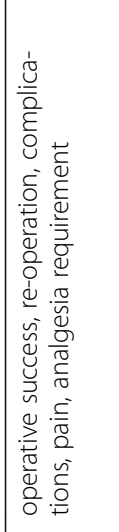 & 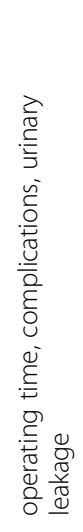 & 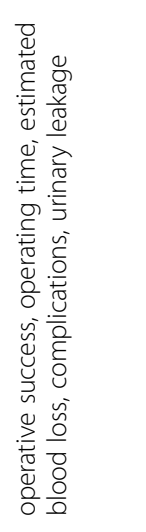 & 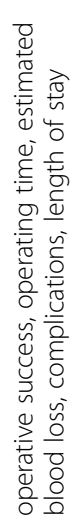 & 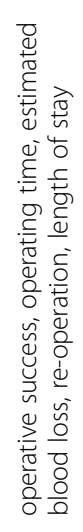 & 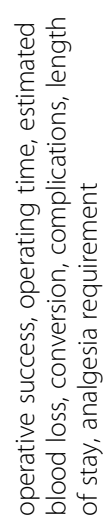 & 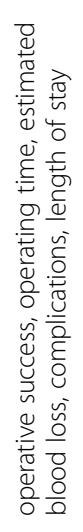 & 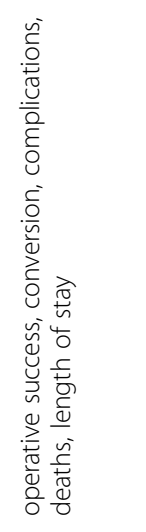 & 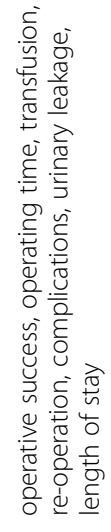 & 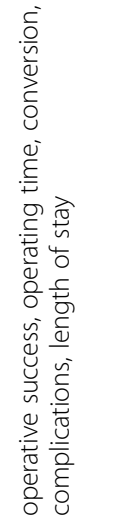 & 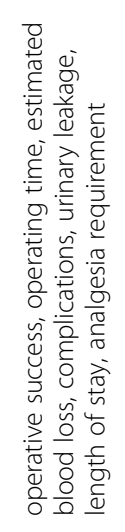 & 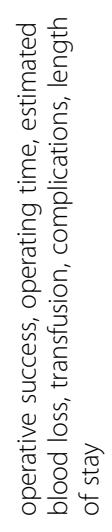 & 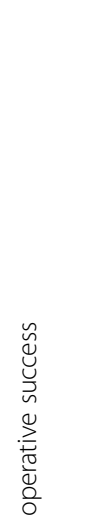 \\
\hline 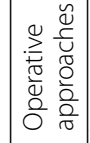 & $\begin{array}{l}0 \\
0 \\
9\end{array}$ & $\begin{array}{l}\hat{\alpha} \\
\bar{a}\end{array}$ & $\begin{array}{l}\text { वे } \\
\text { à }\end{array}$ & $\begin{array}{l}0 \\
0 \\
9^{-}\end{array}$ & $\begin{array}{l}0 \\
0 \\
\mathbf{y}^{-}\end{array}$ & $\begin{array}{l}\text { वे } \\
\text { ò }\end{array}$ & $\begin{array}{l}a \\
\bar{\alpha} \\
0 \\
0^{-}\end{array}$ & $\begin{array}{l}0 \\
0 \\
0\end{array}$ & $\begin{array}{l}0 \\
\text { 今. } \\
0^{\prime}\end{array}$ & $\begin{array}{l}\text { o } \\
0^{-}\end{array}$ & $\begin{array}{l}\overline{0} \\
\text { 今. }\end{array}$ & $\begin{array}{l}\text { cे } \\
\text { à }\end{array}$ & $\begin{array}{l}\text { or } \\
\text { à } \\
\text { sं }\end{array}$ \\
\hline$\frac{5}{4}$ & 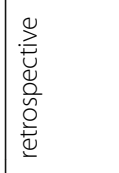 & 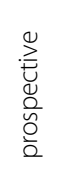 & 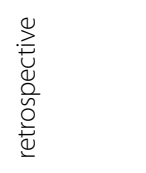 & 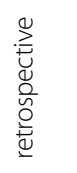 & 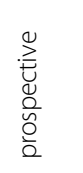 & 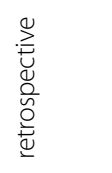 & 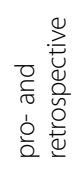 & 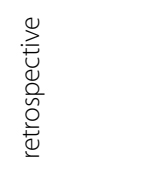 & 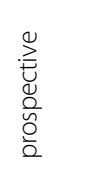 & 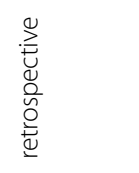 & 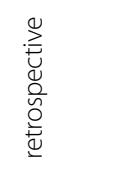 & $\begin{array}{l}\stackrel{0}{Z} \\
\stackrel{u}{0} \\
\frac{0}{0} \\
\frac{0}{2}\end{array}$ & 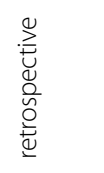 \\
\hline$\frac{0}{0} \frac{0}{2}$ & mo & ঃั & $\stackrel{\sim}{\sim}$ & $\stackrel{\circ}{\sim}$ & $\hat{\bar{n}}$ & ָั & $\stackrel{+}{a}$ & $\stackrel{n}{\grave{n}}$ & ষ্ণ & $\bar{i}$ & $\dot{n}_{\bar{N}}$ & ષ્రి & 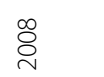 \\
\hline 0 & 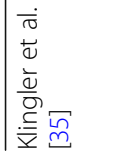 & 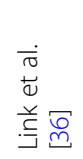 & 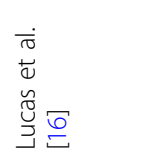 & 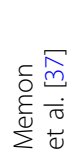 & 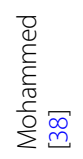 & 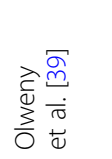 & 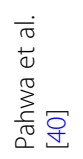 & 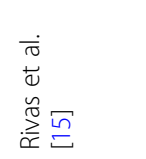 & 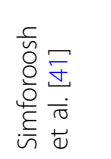 & 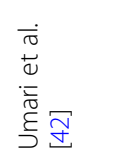 & 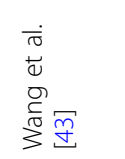 & 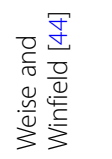 & 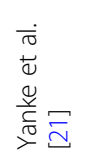 \\
\hline
\end{tabular}




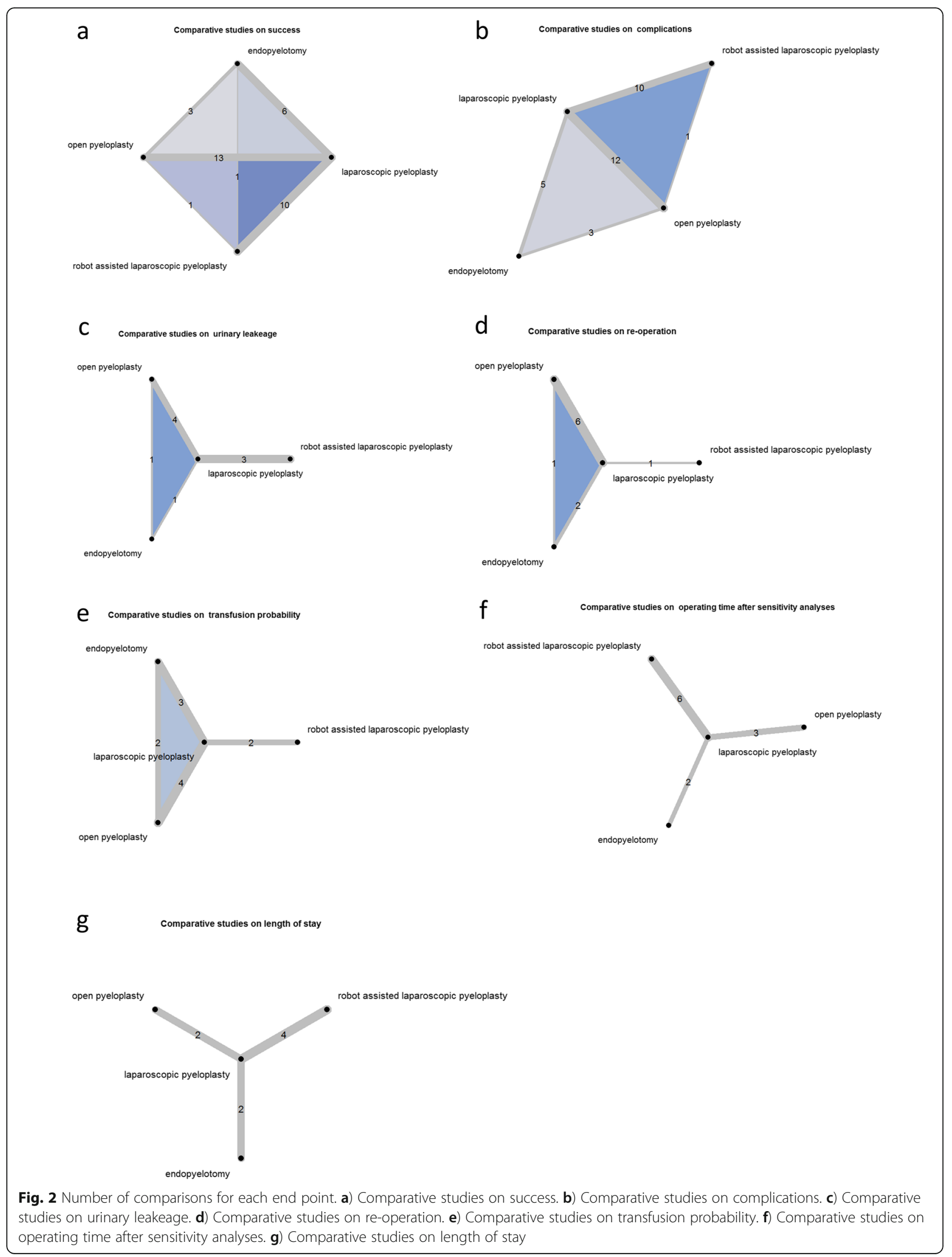




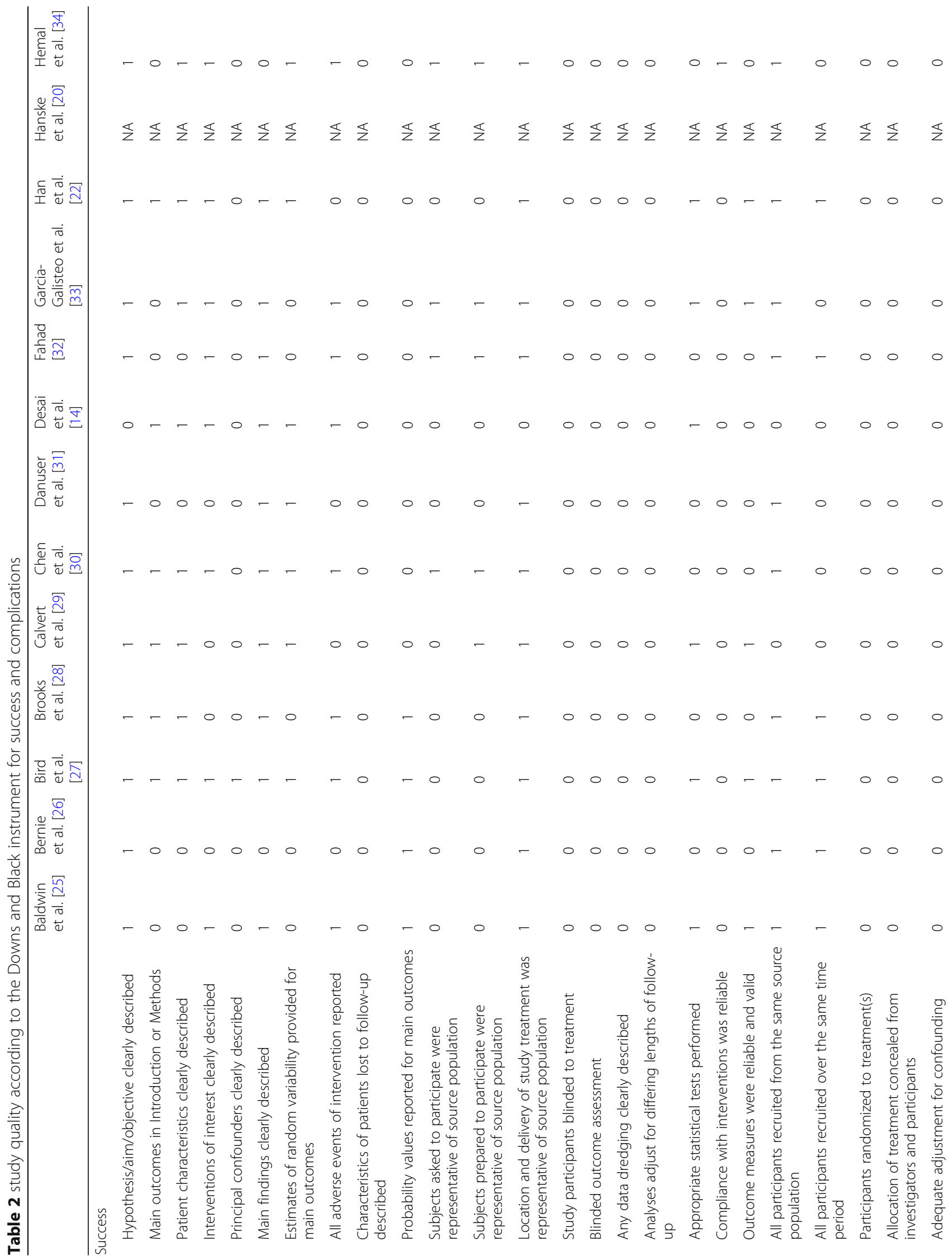




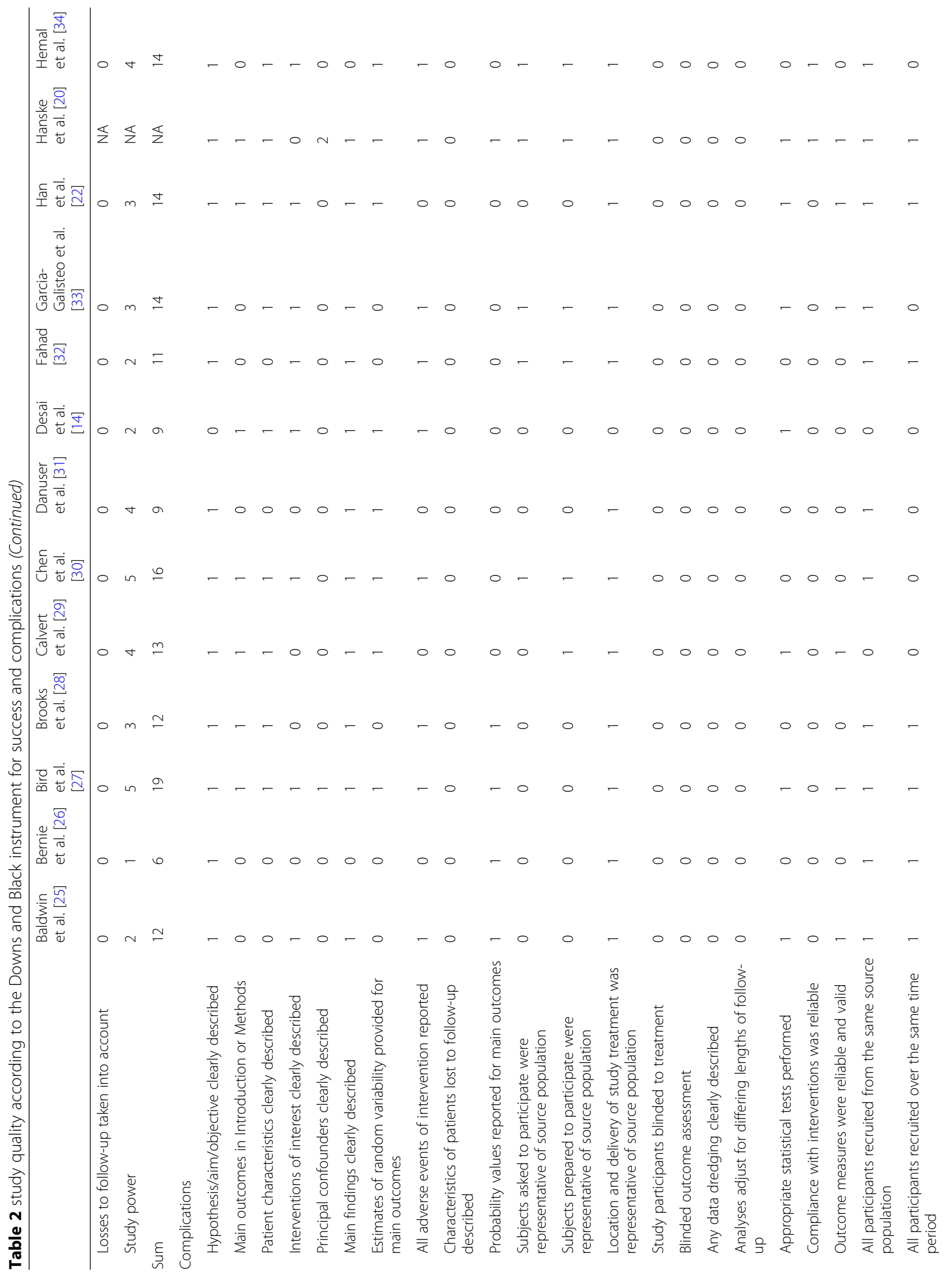




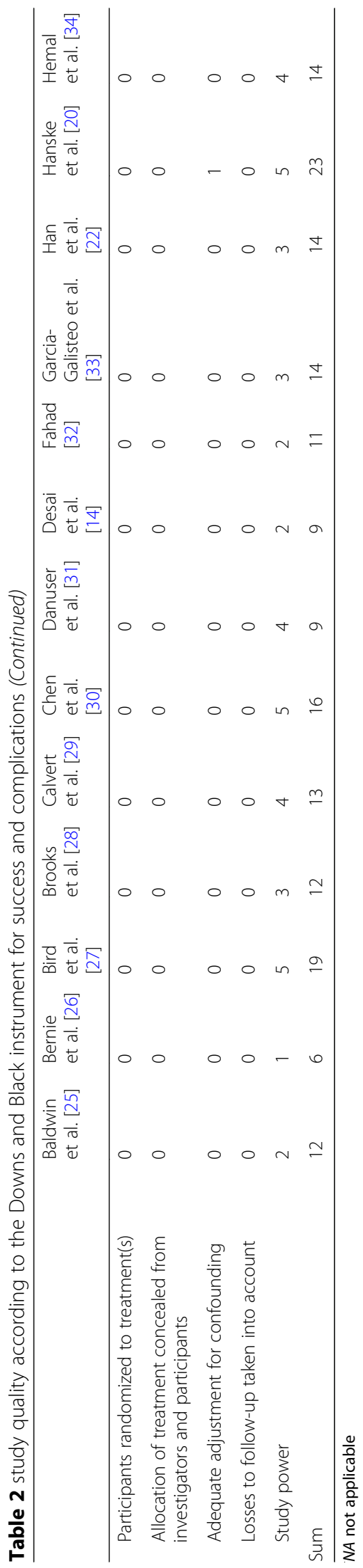




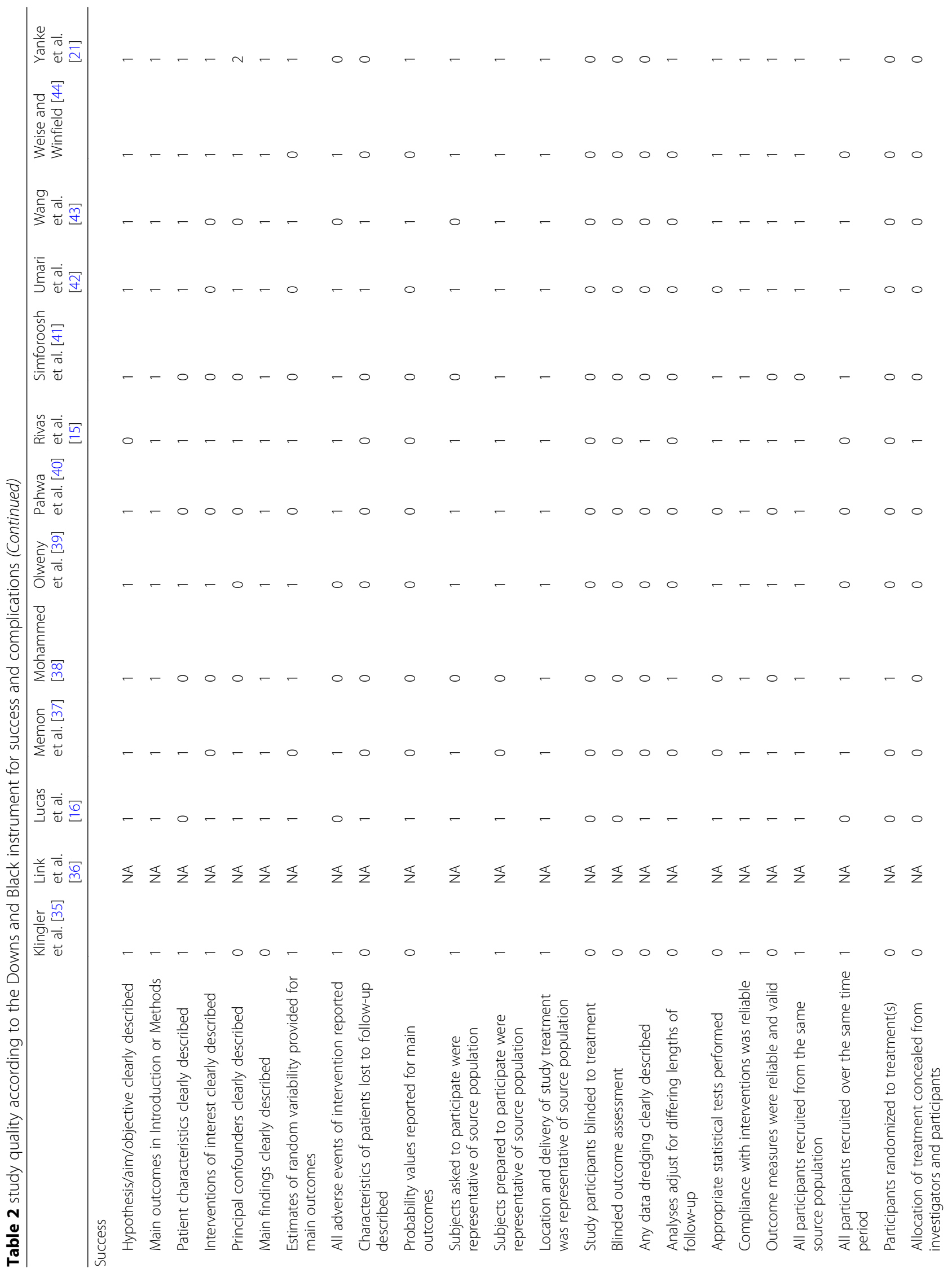




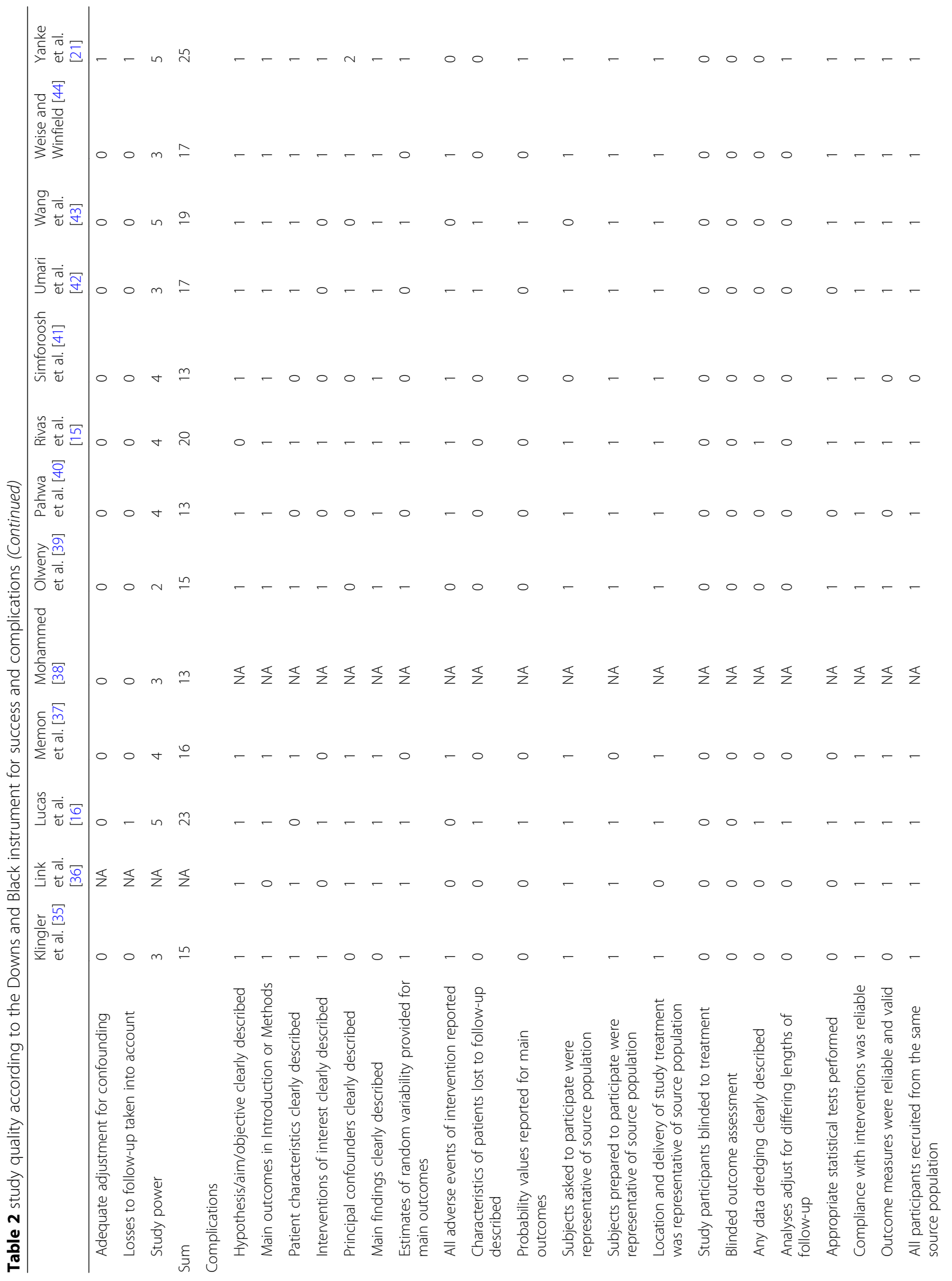




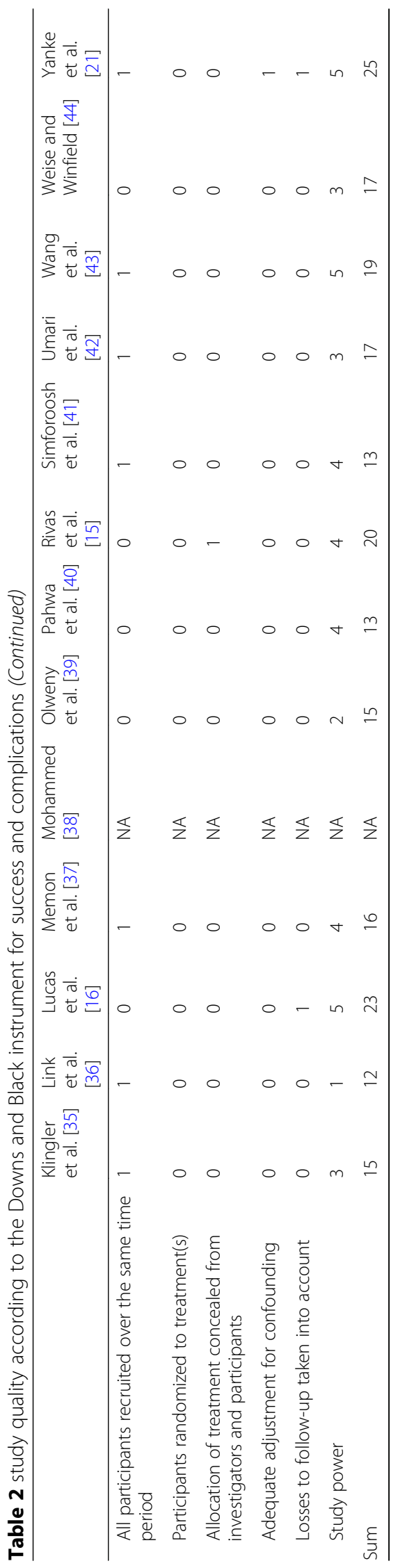




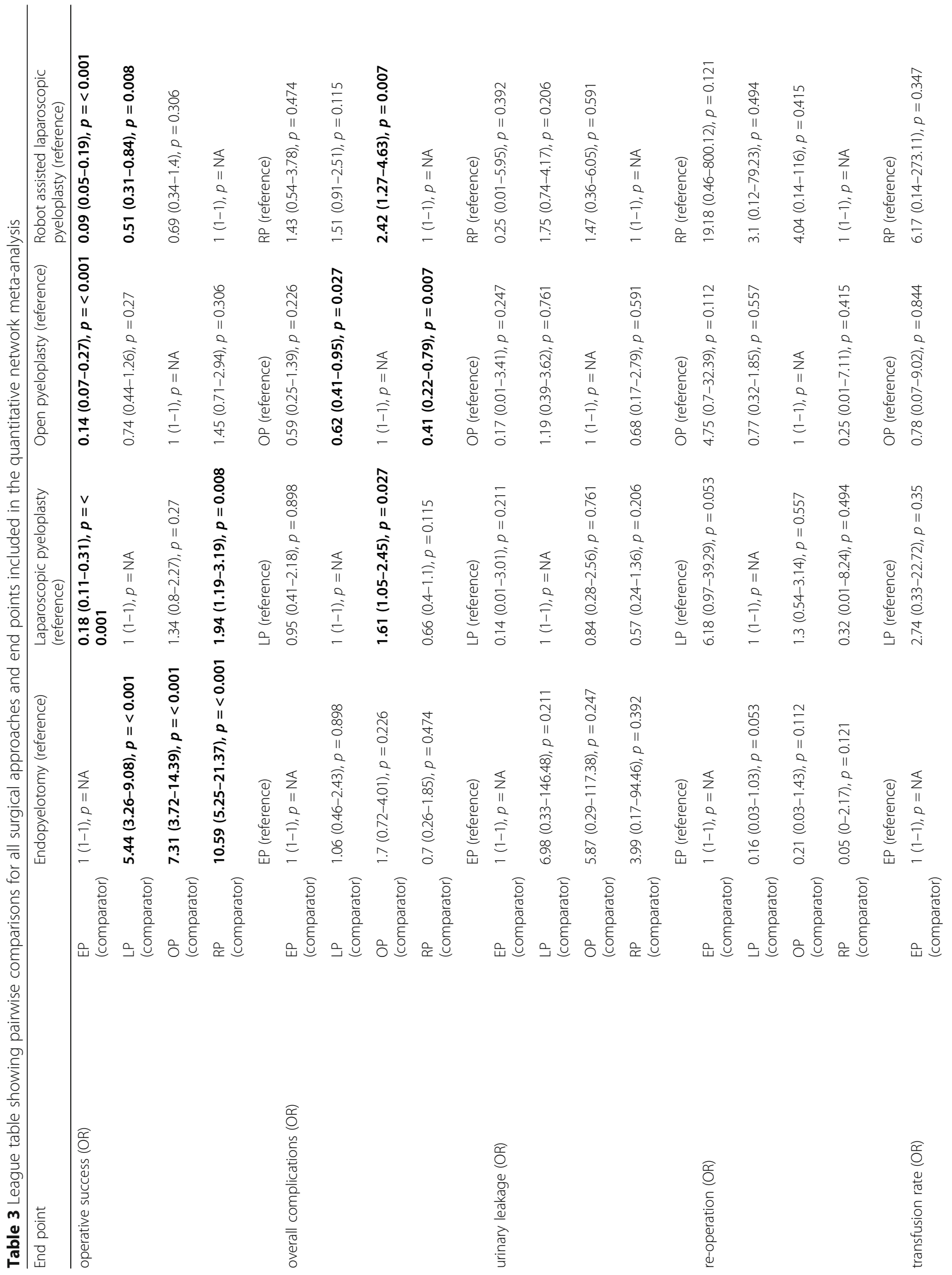




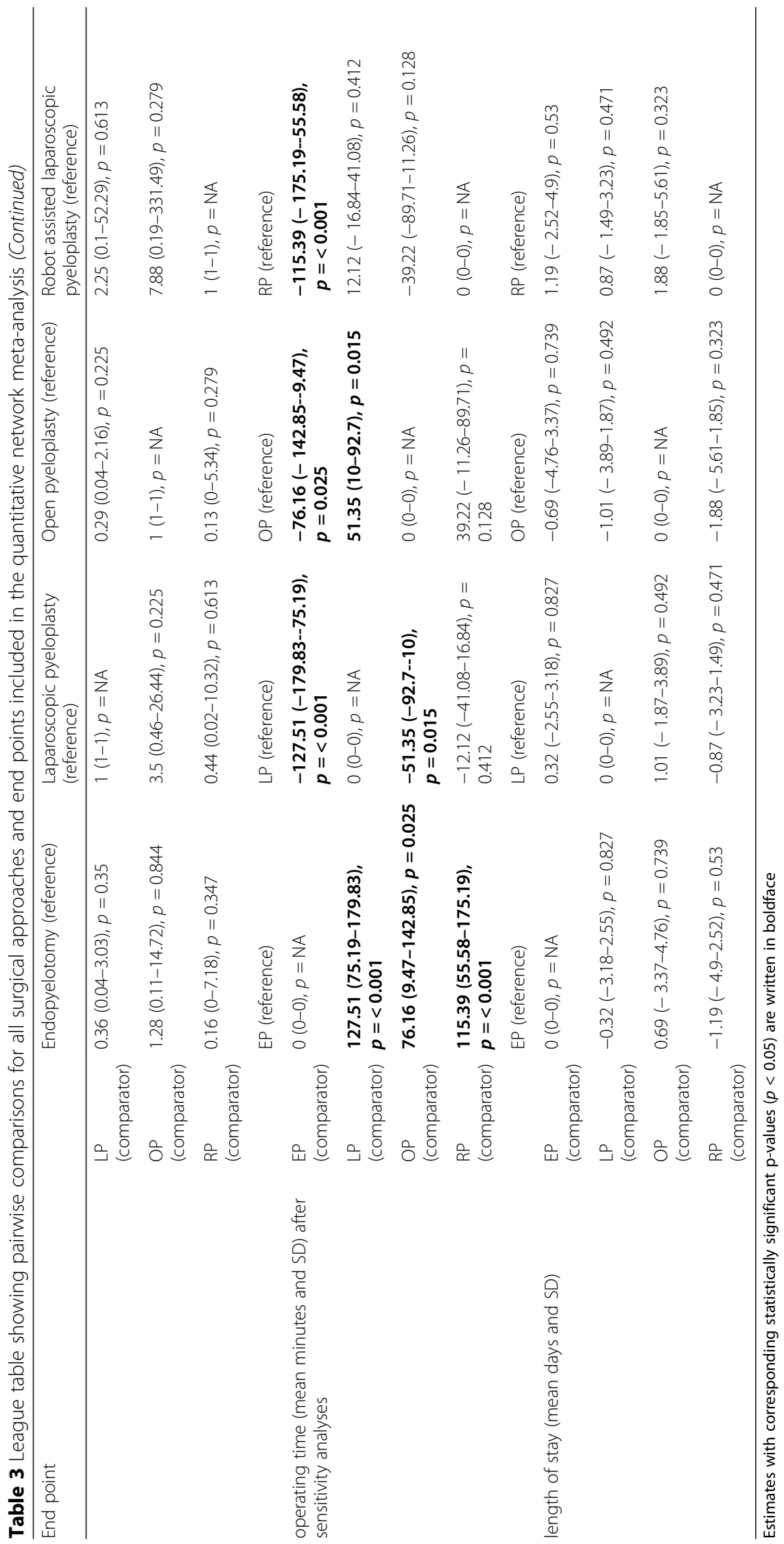


Table 4 P-scores ranking the surgical approaches for every outcome based on the amount of certainty that a single treatment outperforms the average of competing interventions. The p-score ranges from 0 to 1 , the latter indicating the highest certainty possible

\begin{tabular}{|c|c|c|c|c|}
\hline & Endopyelotomy & Laparoscopic pyeloplasty & Open pyeloplasty & $\begin{array}{l}\text { Robot assisted } \\
\text { laparoscopic pyeloplasty }\end{array}$ \\
\hline Operative success & 0.0000 & 0.3797 & 0.6727 & 0.9476 \\
\hline Few overall complications & 0.5583 & 0.4975 & 0.0435 & 0.9007 \\
\hline Few cases of urinary leakage & 0.8584 & 0.1963 & 0.3462 & 0.5991 \\
\hline Low re-operation rate & 0.0476 & 0.6472 & 0.4768 & 0.8284 \\
\hline Low transfusion rate & 0.3089 & 0.6730 & 0.2247 & 0.7934 \\
\hline Short operating time after sensitivity analyses & 0.9958 & 0.0712 & 0.6471 & 0.2860 \\
\hline Short length of stay & 0.4365 & 0.5252 & 0.2589 & 0.7794 \\
\hline
\end{tabular}

was higher among patients with primary UPJO [25]. Calvert et al. reported higher success rates among patients with primary UPJO for LP (98\% vs. 57\%) and OP (96\% vs $67 \%)$ [29].

\section{Network meta-analysis of overall complications}

The network meta-analysis of overall complications included 31 pairwise comparisons from 23 studies. Compared with OP, LP $(\mathrm{OR}=0.62$; 95\%CI 0.41-0.95; $p=0.027)$ as well as RP (OR $=0.41 ; 95 \%$ CI $0.22-0.79 ; p=0.007)$ had a statistically lower risk for complications. No statistically significant difference was detected comparing EP and OP. All pairwise comparisons are depicted in Table 3. Associated p-scores are presented in Table 4 . The study heterogeneity was potentially irrelevant $\left(\mathrm{I}^{2}=13.7 \%, p=0.1416\right)$. Neither Cochran's $\mathrm{Q}(\mathrm{Q}=1.02 ; p=0.9064)$ nor the net heat plot depicted inconsistencies. Figure $3 \mathrm{~b}$ depicts comparisons for OP, RP, LP, and EP.

\section{Network meta-analysis of urinary leakage}

The network meta-analysis of urinary leakage included 9 pairwise comparisons from 7 different studies. Compared with RP none of the other surgical treatment options had a statistically significant higher or lower risk for urinary leakage. Table 3 summarizes these findings, and Table 4 depicts the associated p-scores. There was no evidence of study heterogeneity $\left(\mathrm{I}^{2}=0 \%, p=0.5161\right)$. No inconsistencies were depicted by Cochran's $\mathrm{Q}(\mathrm{Q}=0,58 ; p=0.4471)$ or the net heat plot. Comparisons for OP, RP, LP, and EP as forest-plot are shown in Fig. 3c.

\section{Network meta-analysis of re-operation}

The analysis of re-operation was based on 10 pairwise comparisons from 8 studies. Compared with RP, none of the other surgical treatment options had a statistically significant higher or lower risk for re-operation. All pairwise comparisons are depicted in Table 3. Associated pscores are presented in Table 4. There was no evidence of study heterogeneity $\left(\mathrm{I}^{2}=0 \%, p=0.3001\right)$. Neither Cochran's $\mathrm{Q}(\mathrm{Q}=0.02 ; p=0.9897)$ nor the net heat plot revealed inconsistencies. Figure $3 \mathrm{~d}$ depicts comparisons for OP, RP, LP, and EP. Upon sensitivity analyses including only those studies with at least 12 months follow-up time $(n=13)$, comparable results were obtained and are depicted in Additional file 1: Table S2 [8, 14, 15, 21, 22, $28,30,33-35,38,40,42]$. Five studies stated, whether concomitant stones were present at the time of pyeloplasty but only 2 studies evaluated any effect on one of the outcomes $[15,16,27,28,42]$. Lucas et al. reported that presence of urolithiasis did not affect the rate of secondary interventions [16].

\section{Network meta-analysis of transfusion rate}

The analysis of transfusion rates included 11 pairwise comparisons from 7 studies. Compared with RP none of the other surgical treatment strategies reached a statistically significant difference in transfusion rates as presented in Table 3. Associated p-scores are depicted by Table 4. The study heterogeneity was "moderate" but not statistically significant $\left(\mathrm{I}^{2}=42.3 .7 \%, p=0.4396\right)$. There were statistically significant inconsistencies (Cochran's $Q=6.64, p=0.0361$ ) for which the sources were not identifiable by visual assessment of the net heat plot. Therefore, no sensitivity analyses excluding any studies were possible. Comparisons for OP, RP, LP and EP are depicted by Fig. 3e.

\section{Network meta-analysis of operating time}

A total of 14 pairwise comparisons from 12 studies were included in the analysis of operating time. Compared with EP, RP had a statistically significant longer operating time: mean $=102.87 \mathrm{~min}(95 \% \mathrm{CI} 41.79 \mathrm{~min}-163.95$ min, $p=<0.001)$. Further statistically significant differences in operating times were detected when comparing $\mathrm{LP}$ to EP with mean $=115.13 \mathrm{~min}(95 \% \mathrm{CI} 65.63 \mathrm{~min}-$ $164.63 \mathrm{~min}, p=<0.001)$ and $\mathrm{OP}$ to $\mathrm{EP}$ with mean = $91.96 \mathrm{~min}(95 \% \mathrm{CI} 32.33 \mathrm{~min}-151.58 \mathrm{~min}, p=0.003)$. No statistically significant differences resulted from comparisons of the operative techniques against each other. The study heterogeneity was "considerable" and statistically significant $\left(\mathrm{I}^{2}=95.2 \%, p<0.001\right)$. The net heat plot suggested that the study design comparing EP, LP, and OP 
a: operative success

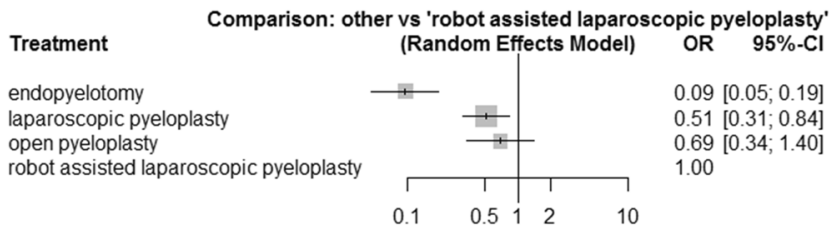

b: complications

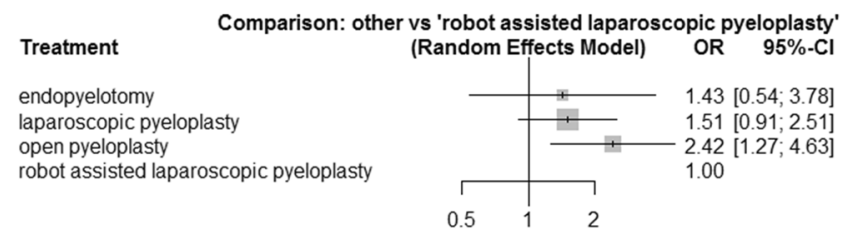

c: urinary leakage

Treatment

Comparison: other vs 'robot assisted laparoscopic pyeloplasty'

endopyelotomy

laparoscopic pyeloplasty

open pyeloplasty

robot assisted laparoscopic pyeloplasty

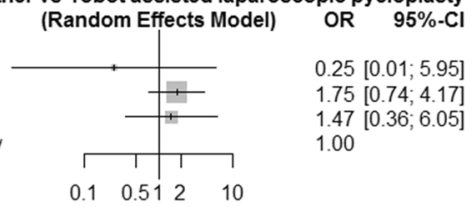

$\mathrm{d}$ : re-operation

Treatment

Comparison: other vs 'robot assisted laparoscopic pyeloplasty'

endopyelotomy

laparoscopic pyeloplasty

open pyeloplasty

robot assisted laparoscopic pyeloplasty

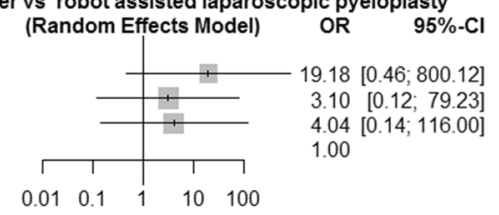

e: transfusion

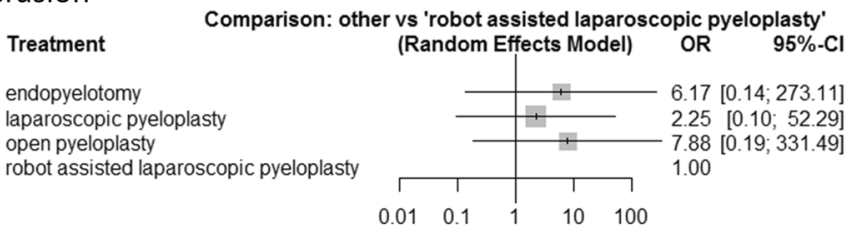

f: operating time after sensitivity analyses

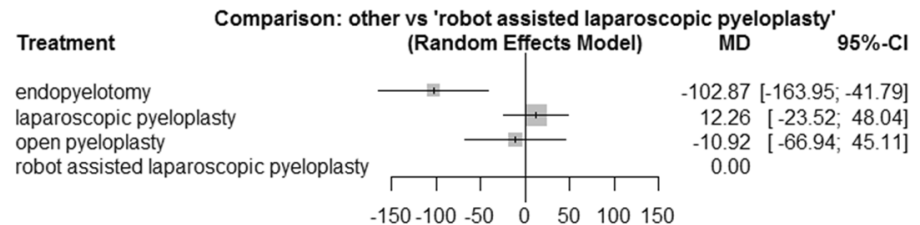

g: length of stay

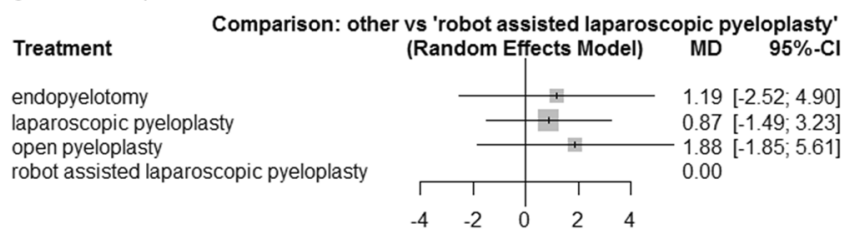

Fig. 3 Pooled estimates for each endpoint. a) operative success. b) complications. c) urinary leakeage. d) re-operation. e) transfusion. f) operating time after sensitivity analyses. $\mathbf{g}$ ) length of stay 
contributed most to network inconsistencies $(\mathrm{Q}=$ 101.59, $p<0.001)$. In consequence, sensitivity analyses excluding the study of Chen et al. were conducted. This resulted in 11 pairwise comparisons from 11 studies. Again, RP had a statistically significant longer operating time when compared to EP mean $=115.39 \mathrm{~min}(95 \% \mathrm{CI}$ $55.58 \mathrm{~min}-175.19 \mathrm{~min}, p=<0.001)$. This was also true for comparisons of LP or OP to EP. For LP to EP with mean $=127.51 \mathrm{~min} \quad(95 \% \mathrm{CI} \quad 75.19 \mathrm{~min}-179.83 \mathrm{~min}$, $p=<0.001)$ and for OP to EP mean $76.16 \mathrm{~min}(95 \% \mathrm{CI}$ $9.47 \mathrm{~min}-142.85 \mathrm{~min}, p=0.025)$. In addition, statistically significant longer operating times resulted from a comparison of LP to OP with mean $=51.35 \mathrm{~min}(95 \% \mathrm{CI} 10$ min-92.7 $\mathrm{min}, p=0.015$ ). Table 3 depicts all pairwise comparisons of the reduced analysis. Associated pscores of the reduced model are detailed in Table 4. The aforementioned "considerable" heterogeneity remained $\left(\mathrm{I}^{\wedge} 2=92.4 \%, p<0.001\right)$ which was not explored further in order to maintain adequate numbers of comparisons. As only direct comparisons remained in the reduced analysis, no evaluation of inconsistencies was reasonable. Bird et al. reported that concurrent treatment of nephrolithiasis did not affect operating time [27]. Figure $3 f$ depicts comparisons for OP, RP, LP, and EP after sensitivity analyses.

\section{Network meta-analysis of length of stay}

The analysis of length of stay included 8 pairwise comparisons from 8 studies. Compared with RP none of the surgical treatment options had a statistically significant shorter or longer length of stay. All pairwise comparisons are depicted in Table 3 whereas Table 4 shows the associated p-scores. Analyses of heterogeneity revealed an $\mathrm{I}^{2}$ of $96.7 \%(p<0.001)$. Due to the available study designs with direct comparisons only, no evaluation of inconsistency was warranted. Again, no further subgroup analyses were performed because of low numbers of comparisons. Comparisons for OP, RP, LP, and EP as forest-plot are shown in Fig. 3g.

\section{Publication bias}

Assessment of publication bias was possible for operative success and complications with the interventions LP versus OP and LP versus RP each. On visual assessment, slight asymmetry was evident for operative success with the comparison of LP versus OP. Less studies reported on ORs $<0.74$. Statistical evaluation did not reveal significant publication bias $(p=0.9118)$. Visual assessment of publication bias for operative success with RP compared to LP showed slight publication bias as well. Less studies reported on ORs $>1.94$. Again, statistical evaluation did not reveal significant publication bias $(p=$ 0.1519). Moreover, visual assessment comparing LP versus OP with respect to complications also resulted in the impression of slight publication bias which was not statistically significant: $p=0.365$. Fewer studies reported on ORs $<0.62$.Visual assessment and statistical evaluation of the comparison of LP and RP yielded no publication bias $(p=0.4808)$. Figure $\mathrm{S} 1$ in the Additional file 1 illustrates the funnel plots.

\section{Narrative meta-analysis of other outcomes}

Only three studies reported on suturing time for RP versus LP [27, 33, 34]. In all cases, suturing time was shorter for RP. Estimated blood loss was reported by 13 studies of which only 4 studies provided estimates of dispersion which did not allow for meta-analyses. In most studies, EP had the lowest blood loss, followed by LP and RP. The highest blood loss was reported for OP in all studies. Due to the nature of the intervention, conversion rates were only reported for RP and LP: Low event rates did not allow for any reliable pooling which applied to death rates as well. Analgesia requirement was reported by 7 studies with different medication such as morphine equivalents, diclophenac, pethidine or tramadol and most of the time without measure of dispersion. Overall, the studies reported EP to have the lowest analgesic requirement, followed by LP and OP. Only one study compared RP and LP and described lower need for analgesic medication for RP. For time to return to normal activity, renal function, and costs only 1 to 3 studies reported estimates with heterogeneous outcome definitions. Therefore, no meta-analysis was possible in these cases.

\section{Discussion}

Several surgical techniques have been developed for treatment of UPJO, each yielding unique advantages and potential limitations. Although RP and LP are allegedly superior, these claims are based on pairwise metaanalyses that failed to evaluate all available techniques at once. In contrast, our study provides a comprehensive overview on OP, EP, LP and RP, comparing their performance with respect to crucial clinical outcomes. Our results indicate that RP is the technique with highest rates of operative success, lowest overall complication rates, shortest hospital stay as well as lowest reoperation rates and transfusion rates. On the other hand, EP yields lowest rates of urinary leakage and shortest operating times. Robot assisted surgery is known for its minimally invasive nature which goes along with less postoperative pain and earlier recovery, which probably causes shorter hospital stays. In addition, robot assisted surgery allows for high precision movements with articulated arms and provides magnified 3-D vision for the surgeon [45]. This might explain high operative success rates of RP. Low transfusion rates of RP are the consequence of the minimally invasive nature of robotic surgery which allows immediate and precise reaction to 
local bleeding [46]. The reason for short operating time in EP probably is that the kidney access requires more time during abdominal surgery than this endoscopic procedure. In addition, EP is less complex, even though the percutaneous approach involves a short flank incision and sewing techniques which are both of low complexity $[47,48]$. Another advantage of EP are low rates of urinary leakage, which might be due to the small extent of manipulation compared to pyeloplasty approaches. However, the reduced invasiveness of EP is on the cost of high recidive rates. Urothelial scarring probably explains these differences since EO only involves an urothelial incision as opposed to surgical approaches where strictured tissue is resected $[49,50]$. When evaluating different treatment approaches, costs have to be taken into account as well. Only one of the included studies evaluated treatment costs [36]: Link et al. reported 2.7 times higher costs for RP (\$5323.80) compared to LP (\$1989.87). More literature is available comparing LP, RP and OP: Yu et al. found RP to be associated with the highest median costs $(\$ 11,829)$, followed by OP (\$9520) and LP (\$8291) [51]. Gettmann et al. published costs for EP ranging between $\$ 3842$ and $\$ 5297$ compared to higher costs for LP (\$7026) and OP (\$7119) [52]. No decision tree analyses have been published to evaluate whether the differences in these expenditures outbalance the benefits of the approaches. Overall study quality was moderate due to limitations in study design such as randomization, concealment of treatment allocation, blinding and omission of multivariable analyses. Still, the nature of interventions and outcomes assessed in this meta-analysis questions, whether higher quality trials would yield relevant changes in the observed effects. Our study is not devoid of limitations, which are mainly inherent to the published trials as its data source: most publications did not adjust for confounding and only one randomized controlled trial could be included. Therefore, the pooled estimates might slightly vary from the true effects. Visual assessment of publication bias yielded minor asymmetries for some the funnel plots. Nevertheless, statistical tests did not return statistically significant evidence for publication bias, which does not completely exclude such bias but suggests low impact. The network meta-analyses of operating time and length of stay yielded statistically significant heterogeneity which could not be bypassed by subgroup analyses in order to maintain adequate numbers of comparisons. Therefore, the pooled estimates might not be generalizable to specific patient subpopulations. Finally, results on the inferiority of EP might be due to differing failure patterns, which is mainly due to missed diagnosis of crossing vessels in EP, and due to inadequate spatulation or incomplete excision of the diseased segment in RP, LP and OP. Still, EP studies were included since they contributed indirect evidence for comparison of other surgical approaches as well. Still, our findings are based on a total of 26 included studies which is the largest meta-analysis published so far and the first comparing more than two interventions simultaneously. The novel network meta-analyses approach further allows for combination of direct and indirect evidence to enhance comparisons of formerly underpowered treatment approaches [53-55].

\section{Conclusions}

Comparing OP, EP, LP and RP for UPJO in a comprehensive network meta-analysis approach, our study found that $\mathrm{RP}$ has the highest rates of operative success and as well as LP lower complication rates than OP. Operating time is shortest for EP, followed by OP, RP, and LP. Surgeons should consider these findings when selecting the optimal treatment method for individual UPJO patients. Further research should aim for improvement of study quality and decision tree analyses based on associated costs.

\section{Supplementary information}

Supplementary information accompanies this paper at https://doi.org/10. 1186/s12894-019-0544-7.

Additional file 1: Figure S1. Funnel plots for operative success and complications with different comparisons Table S1. Operative success based on studies with at least 12 months follow-up $(n=13)$ Table S2. Probability of re-operation based on studies with at least 12 months follow-up $(n=13)$. Table S3. Study quality according to the Newcastle Ottawa Scale.

\section{Abbreviations}

EP: Endopyelotomy; IVU: Intravenous urography; LP: Laparoscopic pyeloplasty; OP: Open pyeloplasty; RP: Robotic assisted pyeloplasty; UPJO: Ureteropelvic junction obstruction

\section{Acknowledgements}

Parts of this paper have been presented as poster presentation at the 2019 European Association of Urology (EAU) conference in Barcelona (poster 1085).

\section{Authors' contributions}

The prespecified title and abstract screening of the EMBASE, MEDLINE and COCHRANE libraries as well as full text assessment were performed by AU, $J U, A v H$ and AS. All other authors performed additional literature screening e.g. of publication lists of reviews and included articles as well as conference proceedings. Data extraction and assessment was performed by all authors. $\mathrm{AU}$ and $J U$ performed all statistical analyses, data interpretation and drafted the manuscript. AS and $\mathrm{AvH}$ contributed technical knowledge with respect to different surgical techniques. LT and MH supervised the study. Data interpretation and drafting of the manuscript were performed by all authors. All authors read and approved the final manuscript.

\section{Funding}

Annemarie Uhlig's work was supported by a Ferdinand Eisenberger Grant of the Deutsche Gesellschaft für Urologie (German Society of Urology), grant ID UhA1/FE-17. We acknowledge financial support for open access publication by the German Research Foundation and the Open Access Publication Funds of the Göttingen University. Both funding sources were uninvolved in data acquisition, analysis, interpretation as well as drafting and revision of the manuscript.

\section{Availability of data and materials}

The extracted study'data and statistical code used for the current study are available from the corresponding author on reasonable request. 


\section{Ethics approval and consent to participate}

Not applicable (meta-analysis).

\section{Consent for publication}

Not applicable (meta-analysis).

\section{Competing interests}

The authors declare that they have no competing interests.

\section{Author details}

'Department of Urology, University Medical Center Goettingen, Goettingen, Germany. ${ }^{2}$ Department of Diagnostic and Interventional Radiology, University Medical Center Goettingen, Goettingen, Germany. ${ }^{3}$ Division of Interventional Radiology, Department of Radiology and Biomedical Imaging, Yale School of Medicine, New Haven, CT, USA. ${ }^{4}$ Department of Thoracic and Cardiovascular Surgery, University Medical Center Goettingen, Goettingen, Germany.

\section{Received: 12 December 2018 Accepted: 24 October 2019} Published online: 11 November 2019

\section{References}

1. Gettman MT, Neururer R, Bartsch G, Peschel R. Anderson-Hynes dismembered pyeloplasty performed using the da Vinci robotic system. Urology. 2002;60:509-13.

2. Schuessler WW, Grune MT, Tecuanhuey LV, Preminger GM. Laparoscopic dismembered pyeloplasty. J Urol. 1993;150(6):1795-9.

3. Mei H, Pu J, Yang C, Zhang H, Zheng L, Tong Q. Laparoscopic versus open pyeloplasty for ureteropelvic junction obstruction in children: a systematic review and meta-analysis. J Endourol. 2011;25(5):727-36.

4. Huang Y, Wu Y, Shan W, Zeng L, Huang L. An updated meta-analysis of laparoscopic versus open pyeloplasty for ureteropelvic junction obstruction in children. Int J Clin Exp Med. 2015;8(4):4922-31.

5. Bansal P, Gupta A, Mongha R, Narayan S, Kundu AK, Chakraborty SC, et al. Laparoscopic versus open pyeloplasty: comparison of two surgical approaches -- a single Centre experience of three years. J Minim Access Surg. 2008:4(3):76-9.

6. Jacobs BL, Kaufman SR, Morgenstern H, Hollenbeck BK, Wolf JS Jr, Hollingsworth JM. Trends in the treatment of adults with ureteropelvic junction obstruction. J Endourol. 2013;27(3):355-60.

7. Khan F, Ahmed K, Lee N, Challacombe B, Khan MS, Dasgupta P. Management of ureteropelvic junction obstruction in adults. Nat Rev Urol. 2014;11(11):629-38.

8. Wang FX, Xu Y, Zhong H. Robot-assisted versus laparoscopic pyeloplasty for patients with ureteropelvic junction obstruction: an updated systematic review and meta-analysis (Provisional abstract). Scand J Urol. 2013;47(4):25164 Available from: http://onlinelibrary.wiley.com/o/cochrane/cldare/articles/ DARE-12013047147/frame.html.

9. Light A, Karthikeyan S, Maruthan S, Elhage O, Danuser H, Dasgupta P. Perioperative outcomes and complications after laparoscopic vs robot-assisted dismembered pyeloplasty: a systematic review and meta-analysis. BJU Int. 2018;122(2):181-94.

10. Cundy TH, Harling L, Hughes-Hallett A, Mayer EK, Najmaldin AS, Athanasiou T, Yang GZ, Darzi A. Meta-analysis of robot-assisted vs conventional laparoscopic and open pyeloplasty in children (Provisional abstract). Database Abstr Rev Eff. 2014;2:582-94 Available from: http://onlinelibrary. wiley.com/o/cochrane/cldare/articles/DARE-12014066171/frame.html.

11. Autorino R, Eden C, El-Ghoneimi A, Guazzoni G, Buffi N, Peters CA, et al. Robot-assisted and laparoscopic repair of ureteropelvic junction obstruction: a systematic review and meta-analysis. Eur Urol. 2014;65(2):430-52.

12. Corbett HJ, Mullassery D. Outcomes of endopyelotomy for pelviureteric junction obstruction in the paediatric population: A systematic review. J Pediatr Urol. 2015;11(6):328-36.

13. Samarasekera D, Chew BH. Endopyelotomy still has an important role in the management of ureteropelvic junction obstruction. Can Urol Assoc J. 2011; 5(2):134-6.

14. Desai MM, Desai MR, Gill IS. Endopyeloplasty versus endopyelotomy versus laparoscopic pyeloplasty for primary ureteropelvic junction obstruction. Urology. 2004;64(1):16-21 discussion.

15. Rivas JG, Gregorio SA, Sanchez LC, Portella PF, Gomez AT, Ledo JC, et al. Evolution in the treatment of the ureteropelvic junction obstruction syndrome. Laparoscopic versus open pyeloplasty. Cent European J Urol. 2015;68(3):384-8.

16. Lucas SM, Sundaram CP, Wolf JS Jr, Leveillee RJ, Bird VG, Aziz M, Pautler SE, Luke P, Erdeljan P, Baldwin DD, Ebrahimi K, Nadler RB, Rebuck D, Thomas R, Lee BR, Boylu U, Figenshau RS, Munver R, Averch TD, Gayed B, Shalhav AL, Gundeti MS, Castle EP, Anderson JK, Duffey BG, Landman J, Okhunov Z, Wong C, Strom KH. Factors That Impact the Outcome of Minimally Invasive Pyeloplasty: Results of the Multi-Institutional Laparoscopic and Robotic Pyeloplasty Collaborative Group. J Urol. 2012;187:522-7.

17. Rothman KJ, Greenland S, Lash TL. Modern epidemiology, vol. x. 3rd ed. Philadelphia: Wolters Kluwer Health/Lippincott Williams \& Wilkins; 2008. p. 758.

18. Downs SH, Black N. The feasibility of creating a checklist for the assessment of the methodological quality both of randomised and non-randomised studies of health care interventions. J Epidemiol Community Health. 1998; 52(6):377-84.

19. Higgins JPT, Green S (editors). Cochrane Handbook for Systematic Reviews of Interventions Version 5.1.0 [updated March 2011]. The Cochrane Collaboration, 2011. Available from www.handbook.cochrane.org.

20. Hanske J, Sanchez A, Schmid M, Meyer CP, Abdollah F, Roghmann F, et al. Comparison of 30-day perioperative outcomes in adults undergoing open versus minimally invasive pyeloplasty for ureteropelvic junction obstruction: analysis of 593 patients in a prospective national database. World J Urol. 2015;33(12):2107-13.

21. Yanke BV, Lallas CD, Pagnani C, McGinnis DE, Bagley DH. The minimally invasive treatment of ureteropelvic junction obstruction: a review of our experience during the last decade. J Urol. 2008;180:1397-402.

22. Han DY, Park KS, Seo IL, Rim JS. A comparison of minimally invasive surgical techniques for ureteropelvic junction obstructions: Endopyelotomy, acucise endopyelotomy, and laparoscopic pyeloplasty. Korean J Urol. 2008;49:592-7.

23. Rucker $\mathrm{G}$, Schwarzer $\mathrm{G}$. Ranking treatments in frequentist network meta-analysis works without resampling methods. BMC Med Res Methodol. 2015;15:58.

24. Egger M, Davey Smith G, Schneider M, Minder C. Bias in meta-analysis detected by a simple, graphical test. BMJ. 1997;315(7109):629-34.

25. Baldwin DD, Dunbar JA, Wells N, McDougall EM. Single-center comparison of laparoscopic pyeloplasty, Acucise endopyelotomy, and open pyeloplasty (Structured abstract). J Endourol. 2003;17(3):155-60 Available from: http:// onlinelibrary.wiley.com/o/cochrane/cleed/articles/NHSEED-22003000812/ frame.html.

26. Bernie JE, Venkatesh R, Brown J, Gardner TA, Sundaram CP. Comparison of laparoscopic pyeloplasty with and without robotic assistance; 2005.

27. Bird VG, Leveillee RJ, Eldefrawy A, Bracho J, Aziz MS. Comparison of robotassisted versus conventional laparoscopic transperitoneal pyeloplasty for patients with ureteropelvic junction obstruction: a single-center study. Urology. 2011;77(3):730-4.

28. Brooks JD, Kavoussi LR, Preminger GM, Schuessler WW, Moore RG. Comparison of open and endourologic approaches to the obstructed ureteropelvic junction. Urology. 1995;46(6):791-5.

29. Calvert RC, Morsy MM, Zelhof B, Rhodes M, Burgess NA. Comparison of laparoscopic and open pyeloplasty in 100 patients with pelvi-ureteric junction obstruction. Surg Endosc. 2008;22(2):411-4.

30. Chen WN, Ye XJ, Liu SJ, Xiong LL, Huang XB, Xu T, Wang XF. Comparison of three surgical methods of ureteropelvic junction obstruction in therapeutic effect and complication. Beijing Da Xue Xue Bao Yi Xue Ban. 2016;48(5):817-21.

31. Danuser H, Stucki P, Pierro GBd, Mattei A. 7 Are there differences in outcome of laparoscopic and robot assisted pyeloplasties and how long is stenting of the anastomosis necessary? J Urol. 2012;187(4S):e3-e.

32. Fahad AH. A comparative study between open and laparoscopic pyeloplasty. J Pharm Sci Res. 2017;9:1961-5.

33. Garcia-Galisteo E, Emmanuel-Tejero E, Navarro Vilchez P, Garcia-Galisteo J, Baena-Gonzalez V. Comparison of the operation time and complications between conventional and robotic-assisted laparoscopic pyeloplasty. Actas Urol Esp. 2011;35(9):523-8.

34. Hemal AK, Mukherjee S, Singh K. Laparoscopic pyeloplasty versus robotic pyeloplasty for ureteropelvic junction obstruction: a series of 60 cases performed by a single surgeon. Can J Urol. 2010;17(1):5012-6.

35. Klingler HC, Remzi M, Janetschek G, Kratzik C, Marberger MJ. Comparison of open versus laparoscopic pyeloplasty techniques in treatment of ureteropelvic junction obstruction. Eur Urol. 2003;44(3):340-5.

36. Link RE, Bhayani SB, Kavoussi LR. A prospective comparison of robotic and laparoscopic pyeloplasty. Ann Surg. 2006;243:486-91. 
37. Memon MA, Biyabani SR, Ghirano R, Aziz W, Siddiqui KM. Is laparoscopic pyeloplasty a comparable option to treat ureteropelvic junction obstruction (UPJO)? A comparative study. J Pak Med Assoc. 2016;66(3):324-7.

38. Mohammed N, Zarzour M, Gadelmoula M, Muhlstadt S, Kawan F, Schumman A, Gollert C, Fornara P. Laparoscopic versus open pyeloplasty in overweight and obese patients. European Urology Supplements. 2017;16(3):e803.

39. Olweny EO, Park SK, Tan YK, Gurbuz C, Cadeddu JA, Best SL. Perioperative comparison of robotic assisted laparoendoscopic single-site (LESS) pyeloplasty versus conventional LESS pyeloplasty. Eur Urol. 2012;61 (2):410-4.

40. Pahwa M, Pahwa AR, Girotra M, Abrahm RR, Kathuria S, Sharma A. Defining the pros and cons of open, conventional laparoscopy, and robot-assisted pyeloplasty in a developing nation. Adv Urol. 2014;2014:850156.

41. Simforoosh N, Basiri A, Tabibi A, Danesh AK, Sharifi-Aghdas F, Ziaee SA, Nooralizadeh A, Hosseini-Moghaddam SM. A comparison between laparoscopic and open pyeloplasty in patients with ureteropelvic junction obstruction. Urol J. 2004;1 (3):165-9.

42. Umari P, Lissiani A, Trombetta C, Belgrano E. Comparison of open and laparoscopic pyeloplasty in ureteropelvic junction obstruction surgery: report of 49 cases. Arch Ital Urol Androl. 2011;83(4):169-74.

43. Wang X, Zhang Z, Peng N, Liu C. Retroperitoneal laparoscopic versus open dismembered pyeloplasty for ureteropelvic junction obstruction. J Xray Sci Technol. 2013;21(3):429-39.

44. Weise ES, Winfield HN. Robotic computer-assisted pyeloplasty versus conventional laparoscopic pyeloplasty. J Endourol. 2006;20(10):813-9.

45. Kim KC. Ohio library and information network. Robotics in general surgery. Online Resource. 2014;xvi:511. https://www.amazon.com/Robotics-GeneralSurgery-Keith-Chae-ebook/dp/BO0GLONSF2.

46. Farnham SB, Webster TM, Herrell SD, Smith JA. Intraoperative blood loss and transfusion requirements for robotic-assisted radical prostatectomy versus radical retropubic prostatectomy. Urology. 2006;67(2):360-3.

47. Novick AC, Gill IS, Klein EA, Rackley R, Ross JH. Operative Urology: Springer Science \& Business Media; 2007.

48. Hofmann R. Endoskopische Urologie: Springer; 2005

49. Hashim H, Woodhouse CRJ. Ureteropelvic junction obstruction. Eur Urol Suppl. 2012;11(2):25-32.

50. Rohrmann D, Snyder HM, Duckett JW, Canning DA, Zderic SA. The operative Management of Recurrent Ureteropelvic Junction Obstruction. J Urol. 1997; 158(3):1257-9.

51. Yu HY, Hevelone ND, Lipsitz SR, Kowalczyk KJ, Hu JC. Use, costs and comparative effectiveness of robotic assisted, laparoscopic and open urological surgery. J Urol. 2012;187(4):1392-8.

52. Gettman M, Lotan Y, Roerhborn CG, Cadeddu JA, Pearle MS. Cost-effective treatment for ureteropelvic junction obstruction: a decision tree analysis. J Urol. 2003:169(1):228-32.

53. Rucker G. Network meta-analysis, electrical networks and graph theory. Res Synth Methods. 2012;3(4):312-24.

54. Caldwell DM, Ades AE, Higgins JP. Simultaneous comparison of multiple treatments: combining direct and indirect evidence. BMJ. 2005:331(7521): 897-900.

55. Mills EJ, Thorlund K, loannidis JP. Demystifying trial networks and network meta-analysis. BMJ. 2013;346:f2914.

\section{Publisher's Note}

Springer Nature remains neutral with regard to jurisdictional claims in published maps and institutional affiliations.

Ready to submit your research? Choose BMC and benefit from:

- fast, convenient online submission

- thorough peer review by experienced researchers in your field

- rapid publication on acceptance

- support for research data, including large and complex data types

- gold Open Access which fosters wider collaboration and increased citations

- maximum visibility for your research: over $100 \mathrm{M}$ website views per year

At $\mathrm{BMC}$, research is always in progress.

Learn more biomedcentral.com/submissions 